\title{
Vibration control of a spinning disk
}

\author{
Jen-San Chen* \\ Department of Mechanical Engineering, National Taiwan University, Taipei 10617, Taiwan
}

Received 22 October 2002; received in revised form 6 October 2003; accepted 13 October 2003

\begin{abstract}
An active control scheme based on the transfer function model is proposed to suppress the vibration of a disk spinning both in the sub- and super-critical speed ranges. The feedback controller consists of a space-fixed point sensor and a point actuator, which are not on any nodal circle of the freely spinning disk. The point sensor can measure both the displacement and the pitching slope of the spinning disk. In the sub-critical speed range, a controller measuring the displacement information alone works well in suppressing the disk vibration. However, such a controller is not only unable to suppress the disk vibration in the super-critical speed range, it may destabilize the spinning disk instead. In order to control a disk in the super-critical speed range, a controller using both the displacement and slope information is proposed. The range of the control gain parameter which renders the spinning disk asymptotically stable can be determined by a stability analysis. The success of such a controller is demonstrated via numerical simulations.

(C) 2003 Elsevier Ltd. All rights reserved.
\end{abstract}

Keywords: Active control; Spinning disk; Critical speed

\section{Introduction}

Spinning disks are the key elements in many mechanical applications, such as gas turbines, grinding wheels, circular saws, and computer disk drives. In general, large amplitude of transverse vibration is undesirable as it can degrade the performance of the devices, and in some cases destroy the machines. For instance, excessive disk vibration degrades the signal from the read/write head in a hard disk drive, and may destroy the disk drive by a head crash. In the wood-cutting industry, the undesirable vibration of the circular saw blade results in unacceptable waste of raw material. In turbine operation, the unstable vibration of the turbine wheel can cause turbine failure due to wheel-to-case impact. These vibration problems are aggravated as industry applications tend to push

* Tel.: +886-2-23661734; fax: +886-2-2-2363-1755.

E-mail address: jschen@ccms.ntu.edu.tw (J.-S. Chen). 


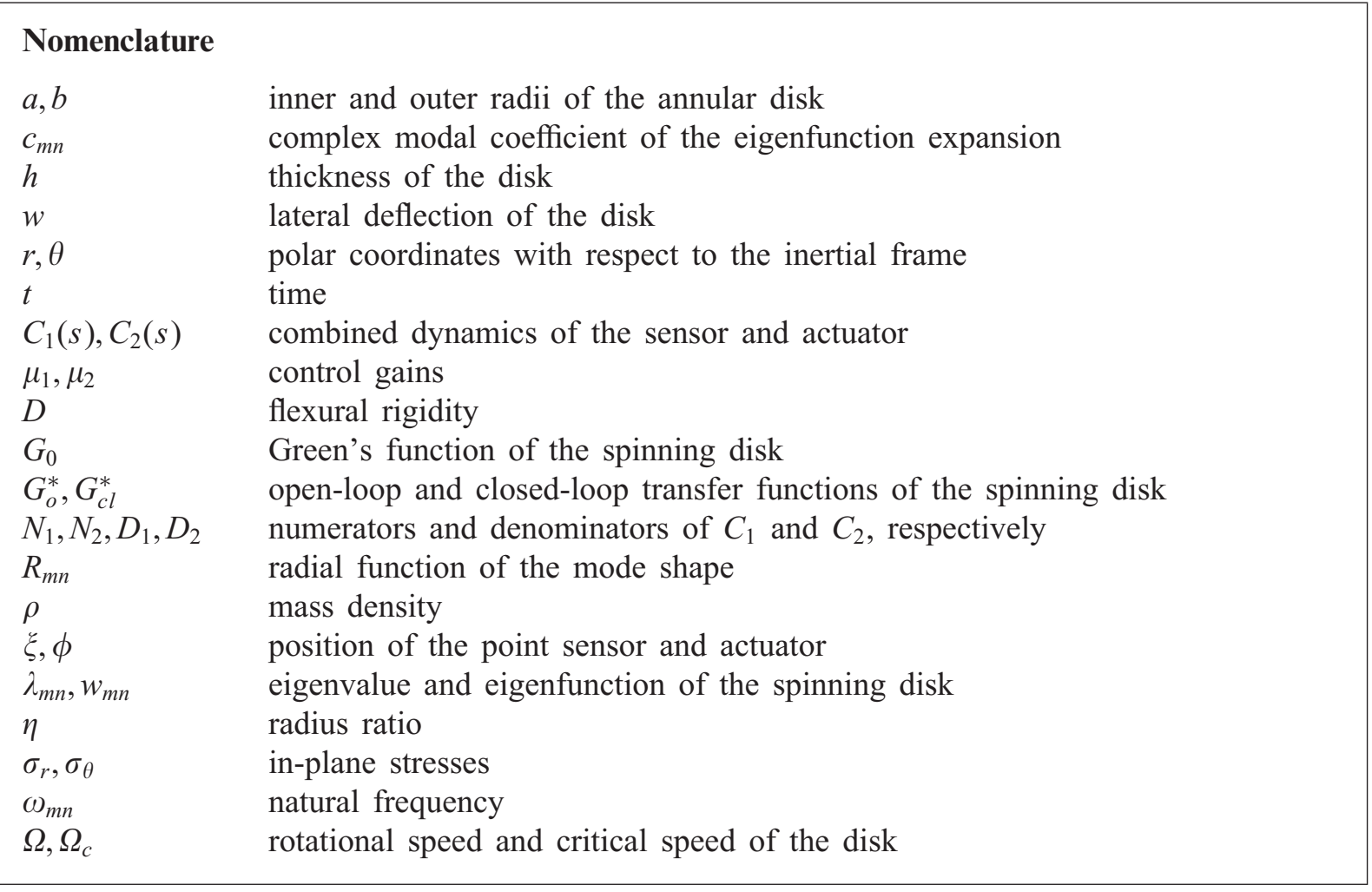

the rotation speed of the circular disk even higher. Suppressing the unwanted vibration in a spinning disk has become an increasing challenge for mechanical engineers and researchers.

One of the most intuitive approaches in preventing excessive vibration in a spinning disk is to apply space-fixed restraints or vibration absorbers on the disk surface. This approach has been widely adopted in circular saw industry [1], and the effect of the space-fixed restraints on the disk vibration has been studied extensively [2-4]. In these analyses, it was found that damping in the space-fixed restraints can suppress the disk vibration when the rotation speed is lower than the critical speed of the disk. However, these passive control approaches fail completely in the super-critical speed range.

In implementing active control to suppress the spinning disk vibration, Ellis and Mote [5] developed a proportional-derivative controller aimed at increasing the transverse stiffness and damping of a circular saw with an electromagnetic actuator. One eddy current sensor close to the actuator is used to measure the displacement. They reported that unstable vibration occurs at high control gain because of complex actuator dynamics which is difficult to accommodate in the controller design. Radcliffe and Mote [6] used the on-line FFT analysis of the disk displacement to identify the dominant mode of the disk vibration. The wave amplitude controller modulates control forces on four electromagnetic actuators to oppose the displacements and velocities predicted by a minicomputer. While this system indeed suppressed the vibration of the controlled $(0,3)$ mode, it excited the uncontrolled $(0,1)$ mode at higher gain due to observation and control spillovers. In order to eliminate the observation and control spillovers, Fung [7] employed the method of independent modal space 
control, a concept originally developed by Meirovitch and $\mathrm{Oz}$ [8] for distributed gyroscopic systems, to suppress the vibration of a disk rotating at varying speeds. With this method, the number of actuators and observers must be equal to the number of modes to be controlled, which may be difficult to implement in practice.

Another approach of active vibration control is to extend the feedback control theory based on lumped system transfer function to the control of distributed systems. Butkovskiy $[9,10]$ described in detail the definition of transfer function of a self-adjoint distributed system in the frequency domain and the associated feedback control theory. Since this approach considers all the vibration modes and the dynamics of actuators and sensors, it can avoid the spillover problems as long as accurate data on the open-loop pole and zero locations can be obtained. Along this line of thought, Kuo and Huang [11] derived the transfer function of a fixed circular disk and proposed several linear controllers based on the root locus arguments.

One of the difficulties in extending the transfer function technique from the self-adjoint systems to the distributed gyroscopic systems is to find the correct transfer functions. This difficulty can be attributed to the lack of orthogonality relations among the eigenfunctions of a gyroscopic system in general. Yang and Mote [12] reformulated the equation of motion of a general distributed gyroscopic system in the first-order state space, and used the orthogonality relations between the eigenvectors of the original and the adjoint systems to derive the transfer function. Based on this transfer function they utilized both the root locus method and the generalized Nyquist criterion to investigate the stability of the closed-loop system. In their investigation they assumed that the stiffness operator was positive definite, which excluded the case of a flexible disk rotating at a super-critical speed.

In this paper, we propose a control scheme which is able to suppress the vibration of a disk spinning both in the sub- and super-critical speed ranges. The control method is based on the transfer function model [10], and requires the measurements of the displacement and pitching slope at a space-fixed point which is not on a nodal circle. First of all we derive the open-loop transfer function of a spinning disk based on the fact that the eigenfunctions of a spinning disk are orthogonal in the physical space, i.e., the second-order space [13]. The transfer function is compared with the one derived by Yang and Mote [12] in the first-order space, and another by Kuo and Huang [11] for a non-rotating disk. The closed-loop transfer function is then derived following a procedure similar to Yang and Mote [12]. Several control laws are examined and one of them is proved to be effective in suppressing the vibration both in the sub- and super-critical speed ranges. By a stability analysis based on the first-order derivatives of the pole positions with respect to the control gain parameters we can determine the range of the control gains which renders the controlled system asymptotically stable. Numerical examples are given.

\section{Open-loop transfer function}

Fig. 1 shows an elastic circular disk clamped on the inner radius $r=a$ and free on the outer radius $r=b$. The disk is spinning with constant rotation speed $\Omega$. We first derive the Green's function of the system. The equation of motion of the spinning disk subject to a space-fixed concentrated impulsive force at position $r=\xi, \theta=\phi$ at time $t=\tau$ can be written in terms of transverse displacement $w$ 


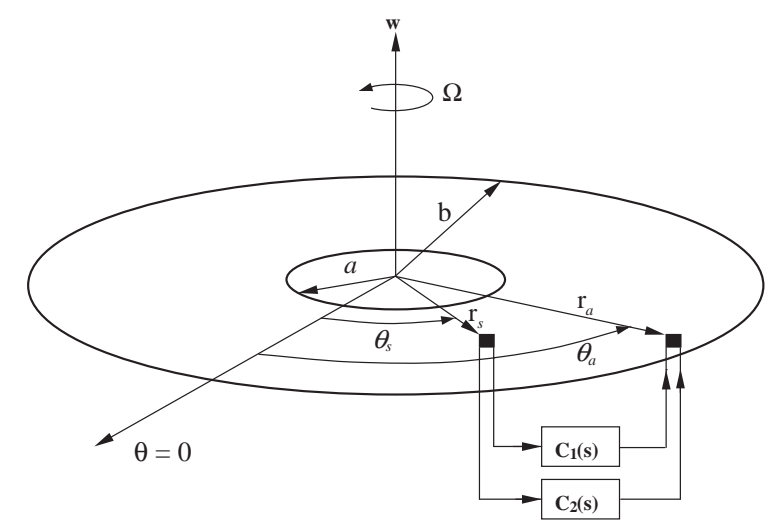

Fig. 1. Schematic diagram of the vibration control of a spinning disk.

and with respect to the space-fixed coordinate system $(r, \theta)$ as

$$
w_{, t t}+2 \Omega w_{, \theta t}+\frac{D}{\rho h} \nabla^{4} w+\left(\Omega^{2}-\frac{\sigma_{\theta}}{\rho r^{2}}\right) w_{, \theta \theta}-\frac{1}{\rho r}\left(\sigma_{r} r w_{, r}\right)_{, r}=\frac{1}{r} \delta(t-\tau) \delta(r-\xi) \delta(\theta-\phi) .
$$

The parameters $\rho, h$, and $D$ are the mass density, thickness, and flexural rigidity of the disk, respectively. The axisymmetric in-plane stresses $\sigma_{r}$ and $\sigma_{\theta}$ are due to the centrifugal effect. $\delta(:)$ is the Dirac delta function. The initial conditions of the spinning disk are assumed to be $w(r, \theta)=w_{0}(r, \theta)$ and $w_{, t}(r, \theta)=v_{0}(r, \theta)$ at time $t=0$.

It is convenient to introduce dimensionless variables (denoted with an asterisk)

$$
\begin{aligned}
& w^{*}=\frac{w}{h}, \quad r^{*}=\frac{r}{b}, \quad \xi^{*}=\frac{\xi}{b}, \quad \eta=\frac{a}{b}, \quad \delta^{*}(\cdot)=b \delta(\cdot), \\
& t^{*}=\frac{t}{b^{2}} \sqrt{\frac{D}{\rho h}}, \quad \tau^{*}=\frac{\tau}{b^{2}} \sqrt{\frac{D}{\rho h}}, \quad \Omega^{*}=\Omega b^{2} \sqrt{\frac{\rho h}{D}}, \quad \sigma_{r}^{*}=\frac{h b^{2}}{D} \sigma_{r}, \quad \sigma_{\theta}^{*}=\frac{h b^{2}}{D} \sigma_{\theta} .
\end{aligned}
$$

After substituting the above dimensionless variables into Eq. (1), and dropping the asterisks in what follows for brevity, Eq. (1) can be rewritten as

$$
w_{, t t}+2 \Omega w_{, \theta t}+\nabla^{4} w+\left(\Omega^{2}-\frac{\sigma_{\theta}}{r^{2}}\right) w_{, \theta \theta}-\frac{1}{r}\left(\sigma_{r} r w_{, r}\right)_{, r}=\frac{1}{r} \delta(t-\tau) \delta(r-\xi) \delta(\theta-\phi) .
$$

For a freely spinning disk, the eigenvalues are purely imaginary and occur in complex conjugate pairs, i.e., $\lambda_{m n}= \pm \mathrm{i} \omega_{m n}$, where $\mathrm{i}=\sqrt{-1}$ and $\omega_{m n}$ is real. The eigenfunction corresponding to $\lambda_{m n}$ is in general complex and assumes the form

$$
w_{m n}=R_{m n}(r) \mathrm{e}^{\mathrm{i} n \theta} .
$$

The eigenfunction corresponding to $\bar{\lambda}_{m n}$ is $\bar{w}_{m n}$, where overbar means complex conjugate. It is noted that the eigenpairs $\left(\lambda_{m n}, w_{m n}\right)$ and $\left(\bar{\lambda}_{m n}, \bar{w}_{m n}\right)$ represent exactly the same physical mode. $w_{m n}$ in Eq. (3) with positive $n$ and positive $\omega_{m n}$ is a backward traveling wave with $n$ nodal diameters and 


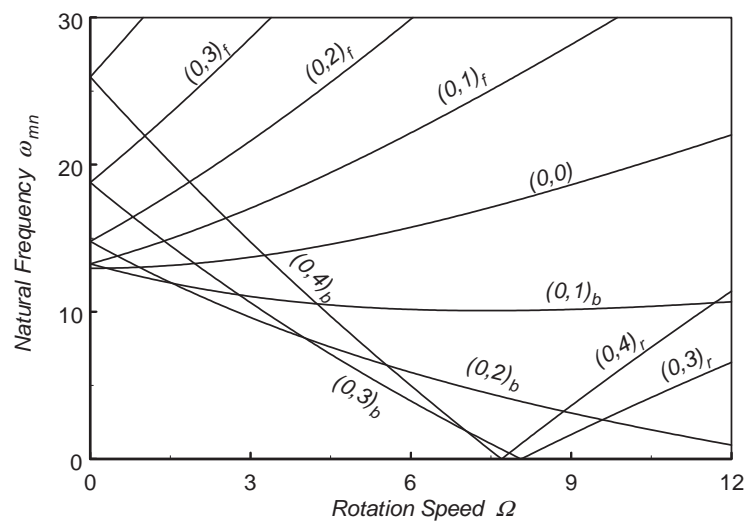

Fig. 2. Natural frequency of a freely spinning disk.

$m$ nodal circles, which is also denoted by $(m, n)_{b}$. Similarly, $w_{m n}$ with negative $n$ and positive $\omega_{m n}$ is a forward traveling wave $(m,-n)_{f}$. The critical speed $\Omega_{c}$ for the mode $(m, n)$ is defined as the rotation speed at which $\omega_{m n}$ of the backward traveling wave $(m, n)_{b}$ becomes zero. For $\Omega$ greater than $\Omega_{c}$, the backward traveling mode appears to travel forward, and is called a reflected wave, denoted by $(m, n)_{r}$. It is noted that for a reflected wave, the integer $n$ is considered as positive, while the natural frequency $\omega_{m n}$ is considered as negative. The radial function $R_{m n}(r)$ can be either solved in a power series form Ref. [14] or can be approximated by the nodal values obtained through a finite element procedure [3]. It is also noted that the eigenfunctions $w_{m n}$ are orthogonal and can be normalized in the following manner [13]:

$$
2 \pi \int_{\eta}^{1} R_{m n}^{2}(r) r \mathrm{~d} r=1 .
$$

Fig. 2 shows the dimensionless natural frequency loci of a spinning disk as the dimensionless rotation speed varies. The radius ratio $\eta$ is 0.5 , and the Poisson ratio is 0.27 . For simplicity, only the modes with less than five nodal diameters are shown here. The natural frequencies of the modes with nodal circle(s) are beyond the range of Fig. 2.

It has been shown in Ref. [15] that the eigenfunctions $w_{m n}$ are complete in the associated Hilbert space. Therefore, the solution $w(r, \theta, t)$ of Eq. (2) can be expressed in terms of $w_{m n}(r, \theta)$ as

$$
w(r, \theta, t)=\sum_{m=0}^{\infty} \sum_{n=-\infty}^{\infty}\left[c_{m n}(t) w_{m n}(r, \theta)\right] .
$$

Both $c_{m n}(t)$ and $w_{m n}(r, \theta)$ in Eq. (5) are complex functions. After substituting Eq. (5) into Eq. (2), multiplying both sides of Eq. (2) by $\bar{w}_{p q}$, and integrating over the annular region we can discretize Eq. (2) into a system of decoupled equations for $c_{m n}(t)$

$$
\ddot{c}_{m n}+2 \mathrm{i} n \Omega \dot{c}_{m n}+\omega_{m n}\left(\omega_{m n}+2 n \Omega\right) c_{m n}=R_{m n}(\xi) \mathrm{e}^{-\mathrm{i} n \phi} \delta(t-\tau) .
$$


A superposed dot represents derivative with respect to time. The initial conditions for $c_{m n}$ are $c_{m n}(0)=$ $c_{m n}^{0}$ and $\dot{c}_{m n}(0)=\dot{c}_{m n}^{0}$, where

$$
c_{m n}^{0}=\int_{0}^{2 \pi} \int_{\eta}^{1} w_{0} \bar{w}_{m n} r \mathrm{~d} r \mathrm{~d} \theta \text { and } \quad \dot{c}_{m n}^{0}=\int_{0}^{2 \pi} \int_{\eta}^{1} v_{0} \bar{w}_{m n} r \mathrm{~d} r \mathrm{~d} \theta .
$$

Recall that at a certain rotation speed there are, in general, two modes with $m$ nodal circles and $n$ nodal diameters. In the sub-critical speed range, one is a forward mode and the other is a backward mode. In the super-critical speed range, one is a forward mode and the other is a reflected mode. In either case, if $\omega_{m n}$ is the natural frequency of one $(m, n)$ mode, $\left(\omega_{m n}+2 n \Omega\right)$ is the natural frequency of the other $(m, n)$ mode, also denoted by $\omega_{m \bar{n}}$. Consequently, Eq. (6) associated with these two modes can be rewritten as

$$
\ddot{c}_{m n}+2 \mathrm{i} n \Omega \dot{c}_{m n}+\omega_{m n} \omega_{m \bar{n}} c_{m n}=R_{m n}(\xi) \mathrm{e}^{-\mathrm{i} n \phi} \delta(t-\tau) .
$$

In other words, $c_{m n}(t)$ associated with these two $(m, n)$ modes are conjugate to each other. Since the mode shapes $w_{m n}$ of these two modes are also conjugate to each other, the contribution of these two modes in Eq. (5) will result in a real displacement $w(r, \theta, t)$.

The solution of Eq. (7) can be written as

$$
\begin{aligned}
c_{m n}(t)= & \frac{H(t-\tau)}{\mathrm{i} \hat{\omega}_{m n}}\left[\mathrm{e}^{\mathrm{i} \omega_{m n}(t-\tau)}-\mathrm{e}^{-\mathrm{i} \omega_{m \bar{n}}(t-\tau)}\right] R_{m n}(\xi) \mathrm{e}^{-\mathrm{i} n \phi} \\
& +\frac{1}{\mathrm{i} \hat{\omega}_{m n}}\left[\mathrm{i} c_{m n}^{0}\left(\omega_{m \bar{n}} \mathrm{e}^{\mathrm{i} \omega_{m n} t}+\omega_{m n} \mathrm{e}^{-\mathrm{i} \omega_{m \bar{n}} t}\right)+\dot{c}_{m n}^{0}\left(\mathrm{e}^{\mathrm{i} \omega_{m n} t}-\mathrm{e}^{-\mathrm{i} \omega_{m \bar{n}} t}\right)\right],
\end{aligned}
$$

where $H(t)$ represents the Heaviside step function, and $\hat{\omega}_{m n}=\frac{1}{2}\left(\omega_{m n}+\omega_{m \bar{n}}\right)$. It is noted that $\hat{\omega}_{m n}$ can be interpreted as the natural frequency of the $(m, n)$ mode as seen by an observer rotating with the disk. After substituting Eq. (8) back into Eq. (5) and rearranging the series, we obtain the response of the spinning disk under concentrated impulse and the prescribed initial conditions as

$$
\begin{aligned}
w(r, \theta, t ; \xi, \phi, \tau)= & G_{0}(r, \theta, \xi, \phi, t-\tau) \\
& +\int_{0}^{2 \pi} \int_{\eta}^{1}\left\{G_{0}(r, \theta, \xi, \phi, t)\left[2 \Omega w_{0, \phi}(\xi, \phi)+v_{0}(\xi, \phi)\right]\right. \\
& \left.+G_{0, t}(r, \theta, \xi, \phi, t) w_{0}(\xi, \phi)\right\} \xi \mathrm{d} \xi \mathrm{d} \phi .
\end{aligned}
$$

$G_{0}$ is the Green's function of the spinning disk

$$
G_{0}(r, \theta, \xi, \phi, t)=\sum_{m=0}^{\infty} \sum_{n=0}^{\infty} \frac{\alpha_{n}}{\hat{\omega}_{m n}} R_{m n}(\xi) R_{m n}(r) \sin \hat{\omega}_{m n} t \cos [n(\theta-\phi-\Omega t)],
$$

where $\alpha_{0}=1$, and $\alpha_{n}=2$ for $n \neq 0$. The Laplace transform of Eq. (10) is the open-loop transfer function of the spinning disk

$$
G_{o}^{*}(r, \theta, \xi, \phi, s)=\sum_{m=0}^{\infty} \sum_{n=0}^{\infty} \frac{\mathrm{e}^{\mathrm{i} n(\theta-\phi)}}{2 \hat{\omega}_{m n}} \alpha_{n} R_{m n}(\xi) R_{m n}(r)\left[\frac{\omega_{m n}}{s^{2}+\omega_{m n}^{2}}+\frac{\omega_{m \bar{n}}}{s^{2}+\omega_{m \bar{n}}^{2}}\right],
$$


where $s$ is a complex parameter. The superposed $(*)$ denotes the Laplace transform of the function. It is noted that Eq. (11) can be reduced to the transfer function derived by Kuo and Huang [11] in the special case of a fixed disk. On the other hand, Eq. (11) cannot be obtained from the expression derived by Yang and Mote [12], in which they assumed that the eigenfunctions in the first-order state space are complete.

For a fixed disk, the transfer function is symmetric about the load point in the circumferential direction, and as a consequence the skew-symmetric part of the response is not controllable. This phenomenon may also be attributed to the fact that each of the eigenvalues of the modes with nodal diameter(s) are degenerate with multiplicity 2, and the minimum number of point actuators required to control the fixed disk is two [16]. When the disk rotates, these degenerate modes split into forward and backward modes and the degeneracy disappears. As a consequence, the minimum number of point actuators required to control the fixed disk is one, except at some special rotation speeds at which the eigenvalue of a backward mode coincides with that of a forward mode. In this special case, the minimum number of point actuators required is two. In this paper, we exclude this special case and assume that the point actuator is not on any of the nodal circles.

\section{Closed-loop transfer functions}

To form a feedback control system, we add a control module to the feedback channel. The feedback control module measures the displacement $w$ and the pitching slope $w_{, \theta}$ at a space-fixed point $\left(r_{s}, \theta_{s}\right)$ and exerts a control force at point $\left(r_{a}, \theta_{a}\right)$, as indicated schematically in Fig. 1 . By using Eqs. (9) and (11), we can write the Laplace transform of the response of the controlled spinning disk as

$$
\begin{aligned}
w^{*}(r, \theta, s)= & G_{o}^{*}\left(r, \theta, r_{a}, \theta_{a}, s\right)\left[\mu_{1} C_{1}(s) w^{*}\left(r_{s}, \theta_{s}, s\right)+\mu_{2} C_{2}(s) w_{, \theta}^{*}\left(r_{s}, \theta_{s}, s\right)\right] \\
& +\int_{0}^{2 \pi} \int_{\eta}^{1}\left\{G_{o}^{*}(r, \theta, \xi, \phi, s)\left[2 \Omega w_{0, \phi}(\xi, \phi)+s w_{0}(\xi, \phi)+v_{0}(\xi, \phi)\right]\right\} \xi \mathrm{d} \xi \mathrm{d} \phi .
\end{aligned}
$$

Transfer functions $C_{1}(s)$ and $C_{2}(s)$ represent the combined dynamics of the sensor, actuator, and the control law for displacement and pitching slope feedbacks, respectively. $\mu_{1}$ and $\mu_{2}$ are the respective control gains. By substituting $(r, \theta)$ by $\left(r_{s}, \theta_{s}\right)$ in Eq. (12) we have the relation

$$
\begin{gathered}
w^{*}\left(r_{s}, \theta_{s}, s\right)\left[1-\mu_{1} C_{1}(s) G_{o}^{*}\left(r_{s}, \theta_{s}, r_{a}, \theta_{a}, s\right)\right]-w_{, \theta}^{*}\left(r_{s}, \theta_{s}, s\right) \mu_{2} C_{2}(s) G_{o}^{*}\left(r_{s}, \theta_{s}, r_{a}, \theta_{a}, s\right) \\
=\int_{0}^{2 \pi} \int_{\eta}^{1}\left\{G_{o}^{*}(r, \theta, \xi, \phi, s)\left[2 \Omega w_{0, \phi}(\xi, \phi)+s w_{0}(\xi, \phi)+v_{0}(\xi, \phi)\right]\right\} \xi \mathrm{d} \xi \mathrm{d} \phi .
\end{gathered}
$$

We can also differentiate Eq. (12) with respect to $\theta$ and substitute $(r, \theta)$ by $\left(r_{s}, \theta_{s}\right)$ again to obtain the relation

$$
\begin{gathered}
-w^{*}\left(r_{s}, \theta_{s}, s\right) \mu_{1} C_{1}(s) G_{o, \theta}^{*}\left(r_{s}, \theta_{s}, r_{a}, \theta_{a}, s\right)+w_{, \theta}^{*}\left(r_{s}, \theta_{s}, s\right)\left[1-\mu_{2} C_{2}(s) G_{o, \theta}^{*}\left(r_{s}, \theta_{s}, r_{a}, \theta_{a}, s\right)\right] \\
=\int_{0}^{2 \pi} \int_{\eta}^{1}\left\{G_{o, \theta}^{*}(r, \theta, \xi, \phi, s)\left[2 \Omega w_{0, \phi}(\xi, \phi)+s w_{0}(\xi, \phi)+v_{0}(\xi, \phi)\right]\right\} \xi \mathrm{d} \xi \mathrm{d} \phi .
\end{gathered}
$$


From Eqs. (13) and (14) we can solve for $w^{*}\left(r_{s}, \theta_{s}, s\right)$ and $w_{, \theta}^{*}\left(r_{s}, \theta_{s}, s\right)$, and substitute them back into Eq. (12) to obtain the response of the controlled spinning disk

$$
w^{*}(r, \theta, s)=\int_{0}^{2 \pi} \int_{\eta}^{1}\left\{G_{c l}^{*}(r, \theta, \xi, \phi, s)\left[2 \Omega w_{0, \phi}(\xi, \phi)+s w_{0}(\xi, \phi)+v_{0}(\xi, \phi)\right]\right\} \xi \mathrm{d} \xi \mathrm{d} \phi,
$$

where $G_{c l}^{*}(r, \theta, \xi, \phi, s)$ is the closed-loop transfer function

$$
\begin{aligned}
G_{c l}^{*}(r, \theta, \xi, \phi, s)= & G_{o}^{*}(r, \theta, \xi, \phi, s) \\
& +\frac{G_{o}^{*}\left(r, \theta, r_{a}, \theta_{a}, s\right)\left[\mu_{1} C_{1}(s) G_{o}^{*}\left(r_{s}, \theta_{s}, \xi, \phi, s\right)+\mu_{2} C_{2}(s) G_{o, \theta}^{*}\left(r_{s}, \theta_{s}, \xi, \phi, s\right)\right]}{1-\mu_{1} C_{1}(s) G_{o}^{*}\left(r_{s}, \theta_{s}, r_{a}, \theta_{a}, s\right)-\mu_{2} C_{2}(s) G_{o, \theta}^{*}\left(r_{s}, \theta_{s}, r_{a}, \theta_{a}, s\right)} .
\end{aligned}
$$

We assume that the transfer functions $C_{1}(s)$ and $C_{2}(s)$ can be expressed in the general forms

$$
C_{1}(s)=\frac{N_{1}(s)}{D_{1}(s)} \quad \text { and } \quad C_{2}(s)=\frac{N_{2}(s)}{D_{2}(s)}
$$

where polynomials $N_{1}(s)$ and $D_{1}(s), N_{2}(s)$ and $D_{2}(s)$ have no common roots, respectively. The associated characteristic equation of the closed-loop system is

$$
D_{1}(s) D_{2}(s)-\mu_{1} N_{1}(s) D_{2}(s) G_{o}^{*}\left(r_{s}, \theta_{s}, r_{a}, \theta_{a}, s\right)-\mu_{2} N_{2}(s) D_{1}(s) G_{o, \theta}^{*}\left(r_{s}, \theta_{s}, r_{a}, \theta_{a}, s\right)=0 .
$$

\section{Controller design}

In the case of an uncontrolled disk, i.e., $\mu_{1}=\mu_{2}=0$, the eigenvalues $s_{m n}=\mathrm{i} \omega_{m n}$ are purely imaginary. As $\mu_{1}$ and $\mu_{2}$ increase from zero, the eigenvalues may remain purely imaginary or start to have nonzero real parts. In order for the controlled spinning disk to be asymptotically stable, all its eigenvalues must lie in the left-half $s$-plane. One way to estimate the effect of the feedback control on the changes of the eigenvalues is to calculate the derivatives of the eigenvalues with respect to the control gains. By following the procedure proposed by Yang and Mote [12] we can derive the following derivatives:

$$
\begin{aligned}
& \left.\frac{\partial s_{m n}}{\partial \mu_{1}}\right|_{\mu_{2}=0, \mu_{1} \rightarrow 0^{+}}=\frac{w_{m n}\left(r_{s}, \theta_{s}\right) \bar{w}_{m n}\left(r_{a}, \theta_{a}\right) N_{1}\left(\mathrm{i} \omega_{m n}\right)}{2 \mathrm{i} \hat{\omega}_{m n} D_{1}\left(\mathrm{i} \omega_{m n}\right)}, \\
& \left.\frac{\partial s_{m n}}{\partial \mu_{2}}\right|_{\mu_{1}=0, \mu_{2} \rightarrow 0^{+}}=\frac{w_{m n}\left(r_{s}, \theta_{s}\right) \bar{w}_{m n}\left(r_{a}, \theta_{a}\right) n N_{2}\left(\mathrm{i} \omega_{m n}\right)}{2 \hat{\omega}_{m n} D_{2}\left(\mathrm{i} \omega_{m n}\right)} .
\end{aligned}
$$

In the case when the actuator and the sensor are at the same location, i.e., $\left(r_{s}, \theta_{s}\right)=\left(r_{a}, \theta_{a}\right)$, the first-order approximation of the eigenvalues of the controlled disk is then

$$
s_{m n}\left(\mu_{1}, \mu_{2}\right) \cong \mathrm{i} \omega_{m n}+\frac{R_{m n}^{2}\left(r_{s}, \theta_{s}\right)}{2 \hat{\omega}_{m n}}\left[\frac{\mu_{1} N_{1}\left(\mathrm{i} \omega_{m n}\right)}{\mathrm{i} D_{1}\left(\mathrm{i} \omega_{m n}\right)}+\frac{\mu_{2} n N_{2}\left(\mathrm{i} \omega_{m n}\right)}{D_{2}\left(\mathrm{i} \omega_{m n}\right)}\right] .
$$


Case 1: $\mu_{2}=0$. This is the most intuitive design in which only the transverse displacement of the spinning disk is measured. We can show that it is impossible for the spinning disk rotating in the super-critical speed range to be stabilized asymptotically by this design. Any polynomial of $s$ can be rearranged into the real and the imaginary parts when $s$ is replaced by $\mathrm{i} \omega_{m n}$. The real part is a function of $\omega_{m n}^{2}$, while the imaginary part is a function of $\omega_{m n}$. Therefore, we can write the transfer function $N_{1} / D_{1}$ as

$$
\frac{N_{1}}{D_{1}}=\frac{N_{1 R}\left(\omega_{m n}^{2}\right)+\mathrm{i} N_{1 I}\left(\omega_{m n}\right)}{D_{1 R}\left(\omega_{m n}^{2}\right)+\mathrm{i} D_{1 I}\left(\omega_{m n}\right)} .
$$

As a consequence, the real part of derivative (18) becomes

$$
\operatorname{Re}\left[\frac{\partial s_{m n}}{\partial \mu_{1}}\right]_{\mu_{2}=0, \mu_{1} \rightarrow 0^{+}}=\frac{R_{m n}^{2}\left(r_{s}, \theta_{s}\right)}{2 \hat{\omega}_{m n}}\left[\frac{D_{1 R}\left(\omega_{m n}^{2}\right) N_{1 I}\left(\omega_{m n}\right)-D_{1 I}\left(\omega_{m n}\right) N_{1 R}\left(\omega_{m n}^{2}\right)}{D_{1 R}^{2}\left(\omega_{m n}^{2}\right)+D_{1 I}^{2}\left(\omega_{m n}\right)}\right] .
$$

Recall that $\hat{\omega}_{m n}$ represents the natural frequency of the spinning disk as seen by a disk-fixed observer and is always positive. The term in the bracket is an odd function of $\omega_{m n}$. In the sub-critical speed range, the natural frequencies of both the forward and the backward waves are positive and there is no difficulty in choosing a controller to stabilize both modes at the same time. In the super-critical speed range, however, the natural frequency of the forward wave remains positive while the natural frequency of the reflected wave becomes negative. Therefore, the controller will always destabilize one mode while stabilize the other. For a specific example we choose a velocity feedback controller

$$
N_{1}(s)=-s, \quad D_{1}(s)=1 .
$$

Eq. (22) then becomes

$$
\operatorname{Re}\left[\frac{\partial s_{m n}}{\partial \mu_{1}}\right]_{\mu_{2}=0, \mu_{1} \rightarrow 0^{+}}=-\frac{\omega_{m n} R_{m n}^{2}\left(r_{s}, \theta_{s}\right)}{2 \hat{\omega}_{m n}} .
$$

Therefore, the velocity feedback controller stabilizes both the forward and backward waves in the sub-critical speed range, but destabilizes the reflected waves in the super-critical speed range. This controller is equivalent to adding a damping element in the space-fixed restraint, and its destabilizing effect in the super-critical speed range was first demonstrated by Iwan and Moeller [2]. Formula (24) can also be found in Ref. [17].

Case 2: $\mu_{1}=0$. In this case, only the pitching slope information is used. Following the similar procedure as described in case 1 we can derive the real part of the first-order derivative as

$$
\operatorname{Re}\left[\frac{\partial s_{m n}}{\partial \mu_{2}}\right]_{\mu_{1}=0, \mu_{2} \rightarrow 0^{+}}=\frac{n R_{m n}^{2}\left(r_{s}, \theta_{s}\right)}{2 \hat{\omega}_{m n}}\left[\frac{D_{2 R}\left(\omega_{m n}^{2}\right) N_{2 R}\left(\omega_{m n}^{2}\right)-D_{2 I}\left(\omega_{m n}\right) N_{2 I}\left(\omega_{m n}\right)}{D_{2 R}^{2}\left(\omega_{m n}^{2}\right)+D_{2 I}^{2}\left(\omega_{m n}\right)}\right] .
$$

The bracket term in Eq. (25) is an even function of $\omega_{m n}$. The sign of the derivative is then dependent upon the number $n$. Recall that the number $n$ for a forward wave is negative, while $n$ for a backward or a reflective wave is positive. Therefore, such a controller will always destabilize the spinning disk both in the sub- and super-critical speed ranges. For a specific example, we examine the case when the control force is proportional to the pitching slope, i.e.,

$$
N_{2}(s)=-1, \quad D_{2}(s)=1 .
$$


Eq. (25) becomes

$$
\operatorname{Re}\left[\frac{\partial s_{m n}}{\partial \mu_{2}}\right]_{\mu_{1}=0, \mu_{2} \rightarrow 0^{+}}=-\frac{n R_{m n}^{2}\left(r_{s}, \theta_{s}\right)}{2 \hat{\omega}_{m n}} .
$$

Apparently, this controller always destabilizes the forward modes both in the sub- and the supercritical speed ranges.

Case 3: $\mu_{1} \neq 0$ and $\mu_{2} \neq 0$ : We have shown in case 1 that to control a disk spinning in the sub-critical speed range many common controllers using displacement information alone will suffice. The challenge is to control the disk in the super-critical speed range. Although it is impossible to control the disk in the super-critical speed range with the controllers described in cases 1 and 2 , we can design a controller using both the displacement and slope information to suppress the disk vibration. For instance, we can combine the velocity feedback controller (23) and the slope feedback controller (26). The first-order approximation of the closed-loop eigenvalue is then

$$
s_{m n}\left(\mu_{1}, \mu_{2}\right) \cong \mathrm{i} \omega_{m n}-\frac{R_{m n}^{2}\left(r_{s}, \theta_{s}\right)}{2 \hat{\omega}_{m n}}\left[\mu_{1} \omega_{m n}+\mu_{2} n\right] .
$$

In order for the eigenvalue changes of both the $(m, \pm n)$ modes in the super-critical speed range to be negative real, the control gain parameters must satisfy the following relations:

$$
\left|\frac{\left(\omega_{m n}\right)_{f}}{n}\right|>\frac{\mu_{2}}{\mu_{1}}>\left|\frac{\left(\omega_{m n}\right)_{r}}{n}\right|,
$$

where subscripts $f$ and $r$ denote forward and reflected waves, respectively. Eq. (29) must be satisfied for every pair of $(m, n)$ modes with one of them being a reflected wave. The most convenient choice will be

$$
\frac{\mu_{2}}{\mu_{1}}=\Omega
$$

\section{Numerical examples}

In this section, we demonstrate how the feedback controllers suppress the spinning disk vibration. We consider the same disk as the one considered in Fig. 2. The initial condition of the spinning disk is assumed to be

$$
w_{0}(r, \theta)=0.1(r-\eta)^{2}\left(\theta^{3}-\theta \pi^{2}\right) \quad \text { and } \quad v_{0}(r, \theta)=0
$$

This initial condition contains every vibration mode of the spinning disk. Fig. 3 shows the displacement history at position $(r, \theta)=(0.8,0)$ of the disk spinning freely at $\Omega=9$, a super-critical speed. The numerical simulation is based on a finite element technique and Newmark integration method proposed by Chen and Hsu [18]. The simulation model includes all the modes $(m, n)$ with $m \leqslant 5$ and $n \leqslant 4$, a total of 45 modes. The highly irregular zigzags of the displacement curves can be considered as the superposition of the responses of these 45 modes. From Fig. 2 we know that at this speed there exists two reflected modes $(0,3)_{r}$ and $(0,4)_{r}$. In Fig. 4, we apply the velocity feedback controller (23) with $\mu_{1}=8$ with both the sensor and the actuator at position $(0.8,0)$. We can see that the displacement at the controlling point decreases when the time is less than about 2. 


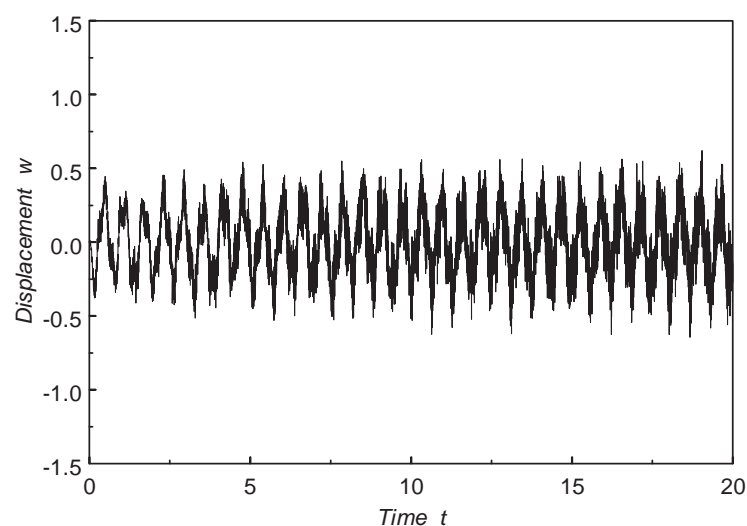

Fig. 3. Displacement history at point $(r, \theta)=(0.8,0)$ of a disk spinning freely at super-critical speed $\Omega=9$.

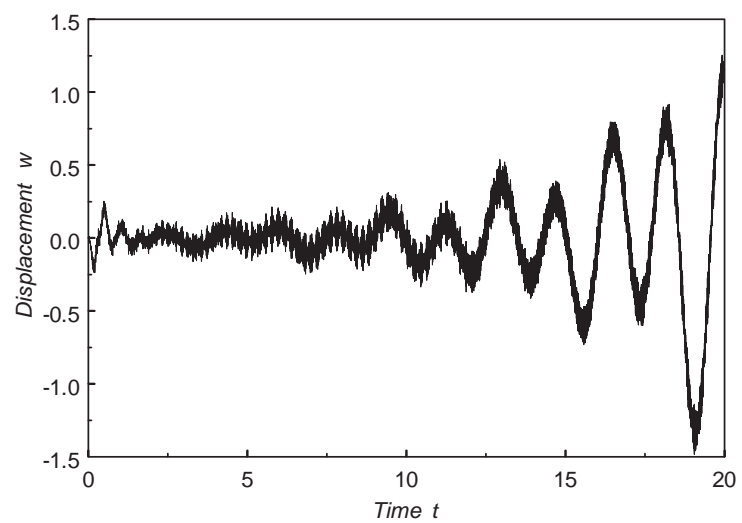

Fig. 4. Displacement history at point $(r, \theta)=(0.8,0)$ of a disk spinning at $\Omega=9$ and under velocity feedback control with $\mu_{1}=8$ at position $(0.8,0)$.

After that the displacement starts to grow exponentially and there are less zigzags along the growing curve. This is because the velocity controller suppresses the vibration of all the non-reflected modes, while it destabilizes the two reflected modes. Therefore, as time goes by the two reflected modes become more and more dominant and the higher-order vibration due to other non-reflected modes dies down eventually. In Fig. 5, we combine the velocity feedback controller (23) and slope feedback controller (26) with $\mu_{1}=8$ and $\mu_{2}=72$ to control the disk spinning at $\Omega=9$. Apparently, the combined controller works well in controlling disk vibration in the super-critical speed range.

When the disk rotates at a sub-critical speed, say, $\Omega=5$, Figs. 6 and 7 show the displacement histories of the freely spinning disk and the one controlled by velocity feedback with $\mu_{1}=8$. We can see that a simple velocity feedback controller is able to suppress disk vibration in the sub-critical speed range. 


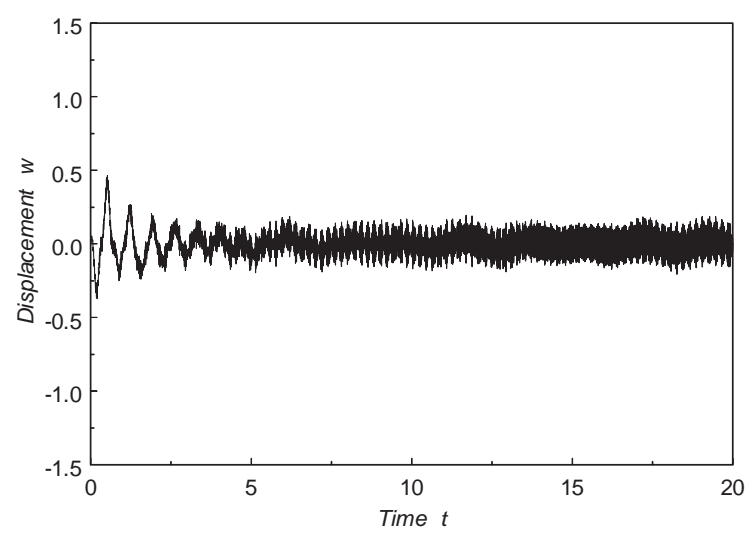

Fig. 5. Displacement history at point $(r, \theta)=(0.8,0)$ of a disk spinning at $\Omega=9$ and under both velocity and pitching slope feedback control with $\mu_{1}=8$ and $\mu_{2}=72$.

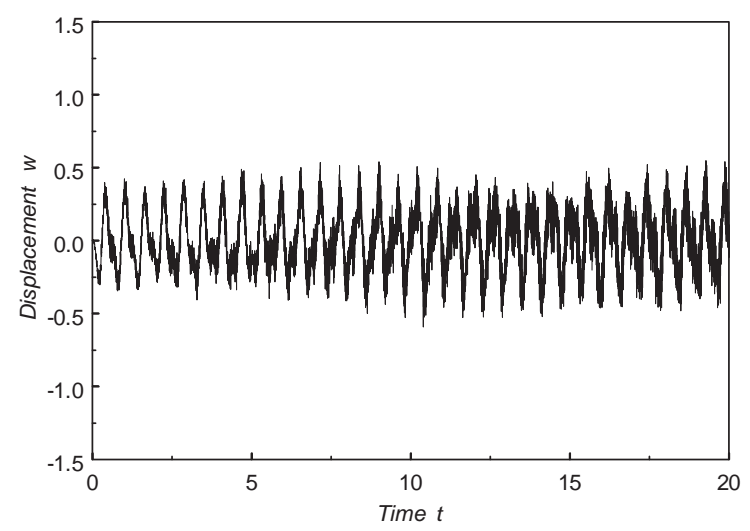

Fig. 6. Displacement history at point $(r, \theta)=(0.8,0)$ of a disk spinning freely at sub-critical speed $\Omega=5$.

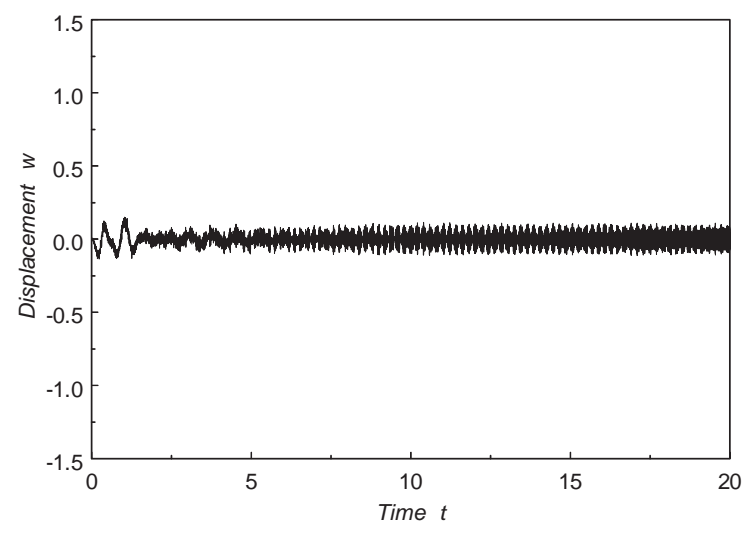

Fig. 7. Displacement history at point $(r, \theta)=(0.8,0)$ of a disk spinning at sub-critical speed $\Omega=5$ and under velocity feedback control with $\mu_{1}=8$. 


\section{Conclusions}

In this paper, we propose an active control scheme based on the transfer function model to suppress the vibration of a disk spinning both in the sub- and super-critical speed ranges. The feedback controller uses both the displacement and pitching slope information at a space-fixed point. Several conclusions can be summarized in the following:

(1) By using the orthogonal relations among the eigenfunctions in the physical space, the transfer function of a spinning disk can be formulated in the second-order space.

(2) The spinning disk is both observable and controllable with a point sensor and a point actuator as long as the sensor and the actuator are not on a nodal circle, and none of the eigenvalues of the freely spinning disk are degenerate.

(3) In the sub-critical speed range, a controller measuring the displacement information alone works well in suppressing the disk vibration. A velocity feedback controller is demonstrated in the numerical example. However, such a controller is not only unable to suppress the disk vibration in the super-critical speed range, it may destabilize the spinning disk instead.

(4) A controller using only the pitching slope information is unable to suppress the disk vibration either in the sub- or super-critical speed range.

(5) In order to control the disk vibration in the super-critical speed range, we need to use a controller which measures both the displacement and the pitching slope simultaneously. The range of the control gain parameters which renders the spinning disk asymptotically stable can be determined by a stability analysis.

\section{References}

[1] Mote Jr CD, Szymani R. Circular saw vibration and control research. Shock and Vibration Digest 1978;10(6): $15-30$.

[2] Iwan WD, Moeller TL. The stability of a spinning elastic disk with a transverse load system. ASME Journal of Applied Mechanics 1976;43:485-90.

[3] Ono K, Chen J-S, Bogy DB. Stability analysis for the Head-Disk interface in a flexible disk drive. ASME Journal of Applied Mechanics 1991;58(4):1005-14.

[4] Sueoka A, Ayabe T, Kurahachi T, Ohishi K. Vibration control of a rotating disk subjected to a periodic external force by dynamic absorbers. JSME International Journal 1992;35(1):41-9.

[5] Ellis RW, Mote Jr CD. Feedback vibration controller for circular saws. ASME Journal of Dynamic Systems, Measurement, and Control 1979;101:44-9.

[6] Radcliffe CJ, Mote Jr CD. Identification and control of rotating disk vibration. ASME Journal of Dynamic Systems, Measurement, and Control 1983;105:39-45.

[7] Fung R-F. Vibration and variable structure control with integral compensation in a non-constant rotating disk system. Journal of Sound and Vibration 1997;199(2):223-36.

[8] Meirovitch L, Oz H. Modal space control of distributed gyroscopic systems. Journal of Guidance and Control 1980;3(2):140-50.

[9] Butkovskiy AG. Green's functions and transfer functions handbook. Chichester, UK: Ellis Horwood Limited; 1982.

[10] Butkovskiy AG. Structural theory of distributed theory. Chichester, UK: Ellis Horwood Limited; 1983.

[11] Kuo CY, Huang CC. Active control of mechanical vibrations in a circular disk. ASME Journal of Dynamic Systems, Measurement, and Control 1992;114:104-12.

[12] Yang B, Mote Jr CD. Frequency-domain vibration control of distributed gyroscopic systems. ASME Journal of Dynamic Systems, Measurement, and Control 1991;113:18-25. 
[13] Chen J-S, Wong C-C. Modal interactions in a spinning disk on a floating central collar and restrained by multiple springs. Journal of the Chinese Society of Mechanical Engineers 1996;17(3):251-9.

[14] Adams G. Critical speeds for a flexible spinning disk. International Journal of Mechanical Sciences 1987;29(8): $525-31$.

[15] Chen J-S, Bogy DB. Mathematical structure of modal interactions in a spinning disk-stationary load system. ASME Journal of Applied Mechanics 1992;59:390-7.

[16] Hughes PC, Skelton RE. Controllability and observability of linear matrix-second-order systems. ASME Journal of Applied Mechanics 1980;47:415-20.

[17] Chen J-S, Bogy DB. Effects of load parameters on the natural frequencies and stability of a flexible spinning disk with a stationary load system. ASME Journal of Applied Mechanics 1992;59(Part 2):230-5.

[18] Chen J-S, Hsu C-M. On the transient response of a spinning disk under a space-fixed load. ASME Journal of Applied Mechanics 1997;64:1017-9. 